\title{
School-based Health Promotion Intervention to Prevent or Delay Type 2 Diabetes in Sub-Saharan Africa
}

\author{
Sophonie Ndahayo* \\ Associate Professor of Public Health, Texila American University, Zambia \\ *Corresponding Author: Sophonie Ndahayo, Associate Professor of Public Health, Texila American University, Zambia.
}

Received: October 17, 2019; Published: November 07, 2019

DOI: $10.31080 /$ ASNH.2019.03.0536

\begin{abstract}
Worldwide rapid social, economic, demographic and cultural transitions particularly in Sub-Saharan Africa has reversed the disease burden from communicable to more complex non-communicable diseases. Type 2 diabetes and its associates is one example of of the health problems ravaging the continent even among children and adolescents. Diabetes is associated with cardiovascular risk factors, including hypertension and hyper- cholesterolaemia, which worsens adverse complications. Other such diseases include overweight and obesity, which are risk-factors for the onset of type 2 diabetes. If left unchecked, diabetes and its complications will take many lives. Therefore, diabetes requires a multisectoral preventive approach that targets the at high-risk groups, including children and adolescents. Studies in the developed world such as clinical trial of a multifaceted school-based intervention suggests that teachers can deliver such an intervention successfully having received training from expert staff. Research authorities such as Pansier and Schulz (2015) and Grey., et al. (2009) found that diabetes is one of the most common chronic childhood diseases; both type 1 and type 2 diabetes are increasing in children globally. Since children and adolescents spend most of the weekly days and hours at school, the school is a critical environment for children with diabetes. This literature review on school-based diabetes interventions and their outcomes demonstrates that since sub-Saharan Africa is leading in non-communicable diseases including type 2 diabetes, the region can adopt this intervention to curb the ever increasing prevalence of type 2 diabetes and its effects for a sustainable development of Sub-Sahara.

Keywords: Risk-Factors; Lifestyle; Prevention; Intervention; Policy and Strategy
\end{abstract}

\section{Introduction and disease burden of the disease}

Type 2 diabetes is a non-communicable disease that is mostly associated with modern lifestyle changes, which include, but not limited to physical inactivity, poor diets and obesity or obesity. Though over the past decades, there has been a lot of medical progress and innovations in the treatment and management of the disease, its prevalence is still increasing at alarming rates. Evidence-based management and prevention strategies are very much needed to prevent the debilitating progression of this disease and costs and losses attached. In the developed world, such as the American states and European countries, school - based diabetes prevention projects have been successfully initiated to promote increased physical activity and healthier eating habits among school children [1]. It is not suprising that schools offer a conducive environment to identify health problems of learners and offer solutions that are most likely to be adhered to. Children pass six to eight hours per working day at school, making it a formal and reliable access point for diabetes risk-factors such as overweight and obesity prevention. In addition, school-going children tend to follow instructions given by the teacher than the parents. Familiarity with parents tend to make the home alone not to bring about the best positive results. The established infrastructure of schools makes school-based intervention efforts one of the most cost-effective methods of preventing childhood obesity [2].

According to Gore., et al. [3], in 2008, the worldwide population of young people aged between 10 and 24 years was more than 1.8 billion, the largest cohort ever, representing $27 \%$ of the population. This number is projected to rise in 2032 to about 2 billion, with $90 \%$ of these people being found in low income and middle-income countries. The size of this population makes their health status of interest, not only as a determinant of future population health, but also for social and economic development. The group becomes of great importance to public health leaders among others who think about sustainable health promotion strategies to prevent and control contemporary health problems among at-risk groups.

It is a fact that though risk factors and the lifestyles that young people adopt might not affect their health during the young age period, they can have a substantial effect in the near future, and can hypothetically affect the health of future generations. For example, high patterns of physical activity that are adopted during childhood and youth, if sustained thereafter, have been proven to have protective effects against the onset of major lifestyle-related health conditions, which including type 2 diabetes. It is because of 
the observation that Gore., et al. [3] stated that preventive strategies should adopt a life-course approach whereby the focus should be on the adolescent and young-adult years.

Considering the facts about diabetes as highlighted by the American Centre for Disease Control (CDC) accessible at www.cdc. gov/diabetes, there is need of a school-based health promotion to prevent type 2 diabetes as it has been found to be threatening even among children, adolescents and young adults. Diabetes is a disease in which the body has either a shortage of insulin, or a decreased ability to use insulin, or both. When diabetes is not controlled, glucose and fats remain in the blood and, over time, damage vital organs (CDC) [4].

Diabetes type 2 can be prevented by application of simple health promoting and disease preventing strategies consisting of healthy food choices; regular and moderate physical activity; and weight loss for those who have more than normal body mass index. Of great concern to public health, in 2010, was that about 1.9 million new cases of diabetes were diagnosed in people aged 20 years or older. This is a group of people that are about or are in universities and soon to be in the working class. If their health status is not improved, great economic losses will be experienced by the countries that invest in their education and start losing on health bills of the same people before paying back to the community.

Satterfield., et al. [5] noted that to control the increasing prevalence of diabetes, public health policies are needed and should focus on prevention strategies to delay or stop the onset of type 2 diabetes. Research studies give new hope for reducing the epidemic of type 2 diabetes with activity for intensive lifestyle adjustments and modest weight control as effective interventions among adults at high risk for developing type 2 diabetes. Strategies such as integration of diabetes education into the school programme and change of the school food supply. Other results pertain to a school programme in which one group was introduced to a curriculum for diabetes prevention, while the other did not. Before and after-tests were administered to determine knowledge of diabetes, foods, exercise, exercise self-efficacy, and related behaviours. The use of the programme showed significant improvements under the following variables: knowledge, self-efficacy and diet, and exercise-related behaviour change.

Considering the above review of literature, and bearing in mind the ever increasing prevalence of diabetes rates, especially in developing countries, taking into account that the world has become a global village: the western lifestyle is lived in middle and low income countries of Sub-Saharan Africa, where the prevalence of diseases of affluence is increasing exponentially, the researcher purposed to highlight the importance of school-based health promotion strategy to prevent or delay diabetes type 2 on the demo- graphically youngest continent Africa, in sub-Saharan Africa specifically where more negative effects are reported.

\section{Risk-factors and epidemiology of diabetes type 2}

According to the World Health Organisation [6], over the last three decades, the prevalence of overweight and obesity has increased substantially. Globally, an estimated 170 million children aged less than 18 years, were then estimated to be overweight. There is childhood overweight in developing countries, and, when taken as group, low-income countries had the lowest prevalence rate. However, overweight has been rising in almost all countries, with prevalence rates growing fastest in lower-middle-income countries. The increased number of overweight and obese peple comes swith serious negative health consequences. The more than enough body weight is a major risk factor for diseases such as cardiovascular disease, type 2 diabetes and many cancers (colorectal cancer, kidney cancer and oesophageal cancer).

It has been estimated that type 2 diabetes prevalence will rise greatly from about 171 million people in 2000 to 366 million people in 2030. Beyond any doubt, preventive strategies and procedures to control or stop the development and progression of this disease were and still are urgently needed [1].

When Alberti., et al. [7] did their research, they said that until recently, type 2 diabetes was typically regarded as a disease of the middle-aged and elderly. While it still was true that this age-group maintains a higher risk than younger adults, evidence has been accumulating that the onset in people aged under 30 years was increasingly observed. Even children and adolescents were then becoming statistics in the diabetes epidemic. Even Alberti., et al. [7] further highlighted that type 2 diabetes had already been reported in children in a number of countries, such as Japan, the United States of America, India, Australia, and the Great Britain. Among children in Japan, type 2 diabetes was already more common than type 1 and accounted for $80 \%$ of childhood diabetes cases.

Globalisation and industrialisation have contributed to the rising prevalence of obesity and type 2 diabetes in children because as part of the effects of sedentary lifestyles, as well as poor eating habits. Future generations may be burdened with morbidity and mortality at the height of their productivity, potentially distressing the workforce and already weak health care systems of countries across the world as alluded to earlier in this paper. This calls for an urgent need to prevent or delay the onset of the diseases even among the young ones [8]. It is encouraging to note that many obesity prevention programmes have utilised environmental modifications in schools, to improve healthy nutrition, increase physical activity, and reduce sedentary behaviour [9]. As low and middle income countries (LMIC) have quickly learnt Western lifestyle prac- 
tices and behaviours, which has brought upon them lifestyle diseases, time has come that LMIC nations apply preventive strategies that their Western world counterparts are applying to control the scourge of non-communicable diseases, including type 2 diabetes.

Reasons for the suggested move are convincing: the global burden of type 2 diabetes is both significant and rising, with most of the increase registered in the last two decades. The largest proportional and absolute increase will occur in developing countries, where the prevalence will rise from 4.2 to $5.6 \%$ [7]. There are ever increasing reports of type 2 diabetes in children worldwide, with some as young as eight years old being affected. By 2030 this will increase to 552 million people representing around $10 \%$ of the global adult population if nothing else is done. Almost $80 \%$ of diabetes deaths occur in low- and middle-income countries and have limited access to affordable treatment. Every eight seconds, somewhere in the world someone dies from diabetes [10]. These statistics should move public health advocates, non-governmental organisations, governments and their partners to act now, when there is still time to prepare future generations for a world free of lifestyle diseases.

In addition to the international Diabetes Federation's, Foster., et al. [11] reiterated that at that time recent data indicated that $16 \%$ of children 6 to 19 years of age in the United States were overweight, and 19\% were obese. Rates are even higher in low and middle income countries, especially among economically disadvantaged groups. Of all the concerns of childhood obesity, the most debilitating is the development of type 2 diabetes.

The need for a policy to create an enabling environment for prevention

World Health Organisation (WHO) [6] reported that obesity (main risk-factor for type 2 diabetes) prevention interventions should be supported by regulations at all levels of governments from the central to community levels. These makes intervention strategies to reach the most vulnerable people and should target the at-risk community groups. Local community tailored interventions result in more cost-effective and results oriented interventions.

Ng., et al. [12] highlighted that from a policy perspective, diabetes can be prevented through school-based interventions, addressing environmental, cultural and economic factors that contribute to obesity.

In addition, Story, Nanney, and Schwartz [13] recognised that schools were identified as a key settings for public health strategies to lower or prevent the prevalence of overweight and obesity, which are risk-factors for type 2 diabetes. These athors further remarked that it also was improbable that childhood obesity rates can be reversed without strong school-based policies and programmes to support healthy eating and physical activity. Stronger policies are needed to provide healthier meals to students at schools; limit their access to low-nutrient, energy-dense foods during the school day; and increase the frequency, intensity, and duration of physical activity at school. There is need of school and state policies. State agencies can impose restrictions on the sale of all unhealthy foods and beverages sold at schools. While these authorities are responsible for the health of the population, it is equally important to inform the reader that our lives our in our hands as far as lifestyle is concerned. We live in a world of human rights, which have twisted the hands of some authorities, including healthcare policies. Some regulations infringe the rights of some groups of people who have the rights to make their choices. Therefore, do not wait for policies enactment and implementation. Decide for yourself and your family members to adopt a health promoting lifestyle, and make choices that prevent diseases, promote health and prolong life.

According to WHO [6], the starting point for policies regarding food and nutrition in a particular nation. Then a policy that inspire food environments and the way in which the dietary guidelines are applied in daily lives of the people. It may be related to government policies on land-use, food manufacturing, marketing, retail, and service. Other examples of policies may include:

- $\quad$ Prohibiting vending machines offering high-calorie, lownutrition foods and beverages such as cola drinks, junk juices, cookies, cake, or other high-fat baked goods

- $\quad$ Schools selling pure still water in place of carbonated soft drinks

- Nutrition education - topic of nutrition and dietary behaviour to be taught at the elementary, middle, and high school levels as part of the health education curriculum (Kann, Telljohann, and Wooley 2007) quoted by Story., et al. [13].

- School-garden programes that provide an opportunity for youth to plant, harvest, and prepare vegetables and some fruits (for example berries, melons, oranges, bananas, etc...) and are frequently linked to the school's academic curriculum (agriculture, biology, nutrition, writing).

- $\quad$ Taking time from physical education and adding it to the academic curriculum

- $\quad$ Staff who teach physical education must have a diploma, undergraduate or graduate training in physical education depending on school level

- Student fitness testing at enrolment time for first year.

- $\quad$ Construction of walking and cycling paths.

Pertaining to physical activity policies, the World Health Organisation recommends that children and adolescents between five and 17 years of age accumulate at least 60 minutes of moderate to vigorous-intensity physical activity every day. For children, physical activity includes play, games, sports, transportation, recreation, physical education, or planned exercise, in the context of family, school and community activities.

\section{Cost-effective preventive methods}

There is now very strong evidence backup both lifestyle intervention and pharmaceutical agents in the prevention of type 
2 diabetes in adults. Nevertheless, the only evidence on diabetes prevention among children focuses on breast-feeding, which may minimize excessive energy intake and improve insulin sensitivity through the higher polyunsaturated fat content. Further pertinent findings have been found in studies of overweight and obese children. For example, in a 20 weeks study of 50 obese adolescents, those randomized to a weight loss programme had, at the end of the study, lower serum insulin levels and blood pressure than those in the control group. These results showed that physical activity improves health and can be used in prevention and control of non-communicalbe diseases, diabetes type 2 included. Physical activity is a cost-effective health promotion intervention that can be practiced especially by young people, and carry the good habit in adulthood, which controls and prevent a good number of chronic diseases.

Good evidence was seen in the Singapore integrated nutrition education programme into the school curriculum. The intervention controlled the school canteens, encouraged water drinking by providing water coolers, targeted obese children for additional assistance, and gave rewarded to schools achieving good health outcomes. Over eight years of the programme (1992 to 2000), the prevalence of obesity fell from 16.6 to $14.6 \%$ in 11-to 12 -year-olds and from 15.5 to $13.1 \%$ in 15 - to 16 -year olds.

Another evidence is the physical exercise intervention in Japan that decreased the prevalence of overweight from 40 to $37 \%$ among boys and to $32 \%$ among girls between the ages of 10 and 13 years, with no change in a control group where there was not physical activity done. A school-based programme among Mexican- American children also has shown the importance of creating a network of social support in the classroom, the home, the school-cafeteria, and among friends and classmates. In comparison to children in a control setting, those in the programme have shown improved physical fitness, reduced numbers with fasting plasma glucose [7]. Foster., et al. [11] reported results of a comprehensive school-based programme, whose results showed a significantly high decrease in several indexes of body fat deposits. These changes may minimise the risk of childhood-onset type 2 diabetes.

School-based health promotion strategy to prevent or delay diabetes type 2

According to Ng, Anderson, McQuillen, and Yu [12], the school setting is an example of a community-based site with enormous potential to address the risk- factors for obesity and type 2 diabetes among children and youth. Baranowski (2002) quoted by Ng., et al. [12] stressed that middle or high-school interventions were more effective than those targeting elementary school students, while Story [13] quoted by Ng., et al. [12] argued that interventions aimed at younger children were more successful than those targeting adolescents. Foster., et al. [11] maintained that schools present openings for reducing the risk of diabetes, since no other establishment has as much contact time with children. It was emphasised that schools can implement environmental changes that affect available foods, physical education, class curricula, and the acceptability of healthy behaviours. Nutrition section can focus on quantity and quality of foods and beverages that are served on campus and around school environments (cafeteria, vending machines, a la carte options, snack bars, school stores, fundraisers, and classroom celebrations). The physical education element can increase the amount of time students spent in physical activity sufficient to raise the heart rate to 130 beats or more per minute or simply put to make the body to sweat. Behavioural knowledge and skills could be transmitted through classroom-based programes. These should be tailored to increase self-awareness, knowledge, behavioural skills such as self-monitoring and goal setting, and peer involvement for behavioural change. Such intervention have been associated with a decrease in the prevalence of obesity, which is a major factor in the development of diabetes.

Nield, Quarrell and Myers [1] also reported results of an intervention to prevent type 2 diabetes, in which the school curriculum included information on diabetes, healthy eating options, and physical activity and fitness testing. The curriculum provided information on the health impacts of diabetes and prevention strategies. The lessons from healthy eating options helped students understand food preparation, body image, healthier snack food options and factors that influence eating habits and behaviour, while the fitness lessons described the benefits of daily physical activity on health outcomes. This is another proof that school-based interventions to prevent diabetes type 2 have been tried and tested and can be replicated where the epidemy is more hampering health and sustainable development of people, especially children and adolescents.

\section{Analysis of the strategy}

Non-communicable diseases (NCDs) in low- and middle-income countries can no longer be ignored or seen as a distraction from the business of prevention and control of infectious diseases [14]. According to WHO [15], up to $80 \%$ of heart disease, stroke, and type 2 diabetes and over a third of cancers could be prevented by eliminating shared risk factors, mainly tobacco use, unhealthy diet, physical inactivity and the harmful use of alcohol. Unless addressed, the mortality and disease burden from these health problems will continue to increase. The World Health Organisation projects that, globally, NCDs deaths will increase by $17 \%$ over the next ten years. The greatest increase will be seen in the African region (27\%) and the Eastern Mediterranean region (25\%). The highest absolute number of deaths will occur in the Western Pacific and South-East Asia regions. Sub-Saharan Africa faces climate change problems related to scare water and poor farming activities. This situation puts the continent predisposes consumers to overweight and obesity - risk-factors to diabetes. It is therefore, pertinent that schools and governments come up with policies to protect pupils and students against risk- factors to type 2 diabetes, which has reached a pandemic level. Policy intervention has been successful in tobacco smoking cessation interventions, thus such strategy can work for type 2 diabetes primordial and primary preventions. 


\section{Conclusion}

Diabetes is currently regarded as a major public health threat in Africa, and, according to the International Diabettes Federation estimates, the number of people with diabetes in Africa will almost double in the 15 years leading up to 2010. The World Health Organisation has set a global objective of reducing death rates due to chronic diseases by two percent a year over the next ten years. If suggested strategies had been followed by all concerned health promotion stakeholders, it could have prevented 36 million premature deaths by 2015 [16]. As children grow into adults, protecting them is a best approach to prevent and control diseases such as type 2 diabetes. Since children and adolescents spend most of the hours of the day at school (6-8 hours), the environment has been recognised to be the ideal for a successful health intervention. Policies that create a good environment will greatly reduce the morbidity and mortality of type 2 diabetes pandemic for a health sustainable sub-Saharan Africa, where the diseases prevalence is exponentially increasing.

\section{Recommendations}

Considering that diabetes has reached an epidemic level and that its prevalence rates will continue to increase, even among the young ones, it is essential that both governments and its partners should urgently enact policies that allow the high-risk groups to prevent, control or delay the onset of diabetes through primordial and primary prevention. As it worked for tobacco-related diseases, diabetes also needs policies that protect children and adolescents who will grow into adults to prevent or delay the onset of the epidemic in Sub-Saharan Africa. As per the reviewed literature, the researcher recommends:

- Health education on non-communicable diseases and diabetes in particular, be integrated into the curriculum of schools at all levels including teacher training schools.

- Schools provide healthier meals to pupils and students

- Limit access to low-nutrient, energy-dense foods during school day

- Increase frequency, intensity and duration of physical activity

- Restriction of sales around schools of unhealthy foods and beverages

- Increase tax of refined foods and soft drinks which are not $100 \%$ juice

- Students' fitness testing at enrolment of first year students/ pupils for follow-up programmes

- Rewarding schools achieving good health outcomes.

\section{Bibliography}

1. Nield A Quarrell S and Myers S. "Community Based Early Intervention for the Prevention of Type 2 Diabetes: A Case Report of the Kahnawake Schools Diabetes Prevention Project" (2013).
2. www.schoolhealthcenters.org

3. Gore FM., et al. "Global burden of disease in young people aged 10-24 years: a systematic analysis". Lancet (2011).

4. www.cdc.gov/diabetes: National centre for chronic disease prevention and health promotion. Diabetes successes and opportunities for population-based prevention and control, at a glance (2011).

5. Satterfield DW., et al. "Community-Based Lifestyle Interventions to Prevent Type 2 Diabetes". Diabetes Care (2003): 9.

6. Population-based approaches to childhood obesity prevention (2012).

7. Alberti G., et al. "Type 2 Diabetes in the Young: The Evolving Epidemic - The International. Diabetes Federation Consensus Workshop". Diabetes Care (2004): 7.

8. www.evidence.nhs.uk

9. Williamson DA., et al. "Effect of an Environmental School-based Obesity Prevention Programme on Changes in Body Fat and Body Weight: A Randomized Trial". 20 (2012): 1653-1661.

10. www.idf.org/webdata/Call-to-Action-on-Diabetes.pdf

11. Foster GD., et al. "School-based intervention for diabetes reduction". New England Journal of Medicine 363 (2010): 443-453

12. Ng C., et al. "School-based Obesity and Type 2 Diabetes Prevention Programs: A Public Health Perspective". Canadian Journal of Diabetes (2005): 211-219.

13. Story M., et al. "Schools and Obesity Prevention: Creating School Environments and Policies to Promote Healthy Eating and Physical Activity". The Milbank Quarterly (2009): 71-100_c.

14. Miranda JJ., et al. "Non-communicable diseases in low- and middle-income countries: context, determinants and health policy". Tropical Medicine and International Health 3 (2009): 1225-1234.

15. 2008-2013 Action plan for global strategy for the prevention and control of non-communicable diseases (2008).

16. www.worlddiabetesfoundation.org

Volume 3 Issue 12 December 2019

(C) All rights are reserved by Sophonie Ndahayo. 